\title{
Probing Stellar Populations in the Outskirts of NGC4244
}

\author{
S. Buehler ${ }^{1}$, A. M. N. Ferguson ${ }^{1}$, M. J. Irwin ${ }^{2}$, N. Arimoto ${ }^{3}$ \\ and P. Jablonka ${ }^{4}$ \\ ${ }^{1}$ Institute for Astronomy, Royal Observatory, Edinburgh \\ email: sbu@roe.ac.uk \\ ${ }^{2}$ Institute of Astronomy, University of Cambridge \\ ${ }^{3}$ NAOJ ${ }^{4}$ Geneva Observatory
}

\begin{abstract}
We are using Subaru/Suprime-Cam to survey the luminous resolved stellar populations in the outskirts of galaxies lying beyond the Local Group. We report here on first results from our analysis of the low mass edge-on galaxy, NGC 4244, lying at $4.4 \mathrm{Mpc}$. Red giant branch stars are clearly resolved in the outer disk and extraplanar regions and our preliminary stellar density maps suggest the presence of an extended and asymmetric stellar component reaching significant distances above the disk plane.
\end{abstract}

\section{Introduction}

The structure and composition of the faintest components of galaxies - stellar halos, thick disks and outer thin disks - contain many important clues for understanding the galaxy assembly process. Numerical simulations of galaxy formation predict that stellar halos and thick disks are a generic feature of galaxies consisting largely of tidal debris (and residual cores) from accreted satellite galaxies (e.g. Bullock \& Johnston 2005). Due to their low surface brightness, observations of stellar halos and thick disks around external galaxies are extremely challenging. Searches based on diffuse emission have yielded a few detections (e.g. Zibetti \& Ferguson 2004) and a few non-detections (e.g. Morrison et al. 1994); it remains unclear if these results reflect real variance in the properties of halos or simply the difficulty of photometering galaxies to ultra-deep surface brightness levels.

An alternative approach to studying the faintest components of galaxies is to use resolved star counts. This method is most successful when applied on large scales, as this allows shallow density and population gradients to be traced and also the discrimination between local density enhancements (i.e. substructure) and any underlying smooth component. This method has now been applied to the Local Group spirals, allowing their structural components to be analysed to surface brightness of $\mathrm{V} \leqslant 30$ mags/ sq arcsec (e.g. Ferguson et al. 2002, Irwin et al. 2005).

We have started a program using Subaru/Suprime-Cam and VLT/VIMOS to image large areas around all massive galaxies lying within $5 \mathrm{Mpc}$. We present first results here for the low mass, edge-on system NGC4244 ( $\mathrm{D}=4.4 \mathrm{Mpc}$ Seth et al. 2005)). Observations for NGC4244 ( $\sim 2 \mathrm{~h}$ exposures in each of the V and I bands) were obtained in January 2005 using Suprime-Cam on Subaru, Hawaii. The seeing was in the range 0.7-0.9". Fig.1, left panel, shows the stacked i-band mosaic of our field.

\section{Preliminary Results}

A clear excess of point-like sources is detected in the vicinity of of NGC 4244 relative to the more distant field. On a colour-magnitude diagram (CMD), these sources also define 

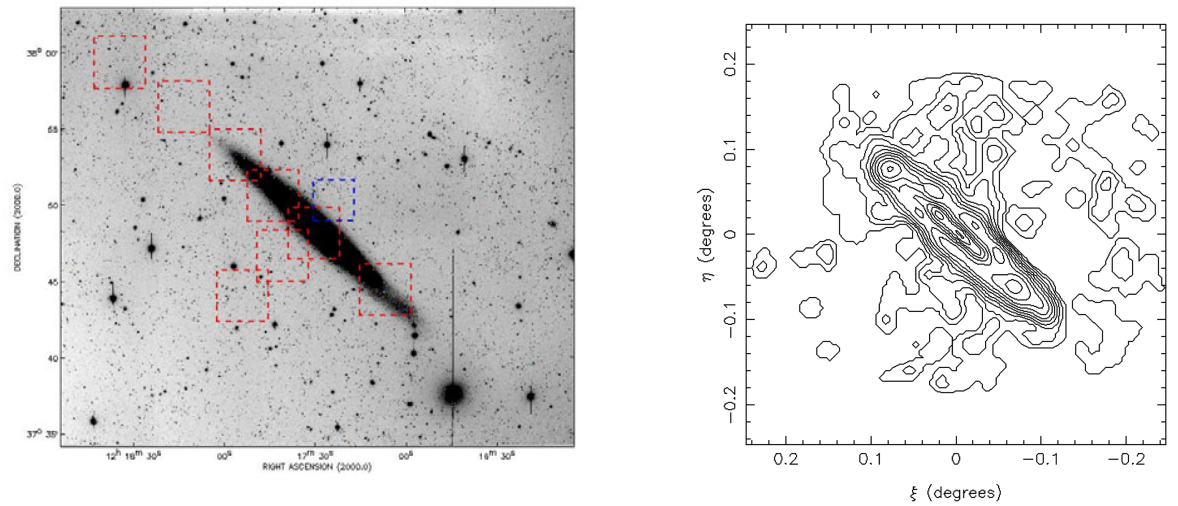

Figure 1. Left: Our I-band mosaic of NGC 4244 with a field size of $33^{\prime} \times 28.5^{\prime}(36.5 \times 31.5$ $\mathrm{kpc}$ at the distance of the galaxy). Right: Contour map of the distribution of point-like sources which have magnitudes and colours appropriate for RGB stars at the distance of NGC4244.

a blue red giant branch $(\mathrm{RGB})$ which reaches to $\mathrm{I} \approx 24$. Indeed, the CMD of extended sources shows no such enhancement in this same region of the CMD.

Figure 1, right panel, shows a contour map of the distribution of RGB stars around NGC 4244 as derived from our stellar density map. The distribution of these sources is clearly extended and asymmetric. RGB stars are detected both above and below the disk plane, reaching to at least $\approx 15 \mathrm{kpc}$ on the north side of the galaxy. Note that our density map of extended sources is essentially featureless.

\section{Discussion and Future Work}

We have successfully detected individual RGB stars and mapped their distribution around NGC4244, lying at $4 \mathrm{Mpc}$. Our preliminary results indicate these stars trace an extended and asymmetric distribution around the galaxy. This irregular appearance suggests the extraplanar stars may be due to a recent accretion rather than a well-settled stellar halo. Fry et al. 1999 obtained deep images of the diffuse light around NGC4244 but did not detect any component beyond the thin exponential disk. However, their detection limit was only $27.5 \mathrm{R}-\mathrm{mag} / \mathrm{sq}$ arcsec. We are currently analysing our data in order to derive the radial surface density and colour profile of the extraplanar stars. Future work will involve conducting similar analyses on the less-inclined spirals NGC 2403 and M81.

\section{References}

Bullock, J. S. \& Johnston, K. V. 2005, ApJ 635, 931

Ferguson, A. M. N., Irwin, M. J., Ibata, R. A., Lewis, G. F., \& Tanvir, N. R. 2002, AJ 124, 1452

Fry, A. M., Morrison, H. L., Harding, P., \& Boroson, T. A. 1999, AJ 118, 1209

Irwin, M. J., Ferguson, A. M. N., Ibata, R. A., Lewis, G. F., \& Tanvir, N. R. 2005, ApJ 628, 105

Morrison, H. L., Boroson, T. A., \& Harding, P. AJ 108, 1191

Seth, A. C., Dalcanton, J. J., \& de Jong, R. S. 2005, AJ 130, 1574

Zibetti, S. \& Ferguson, A. M. N. 2004, MNRAS 352, 6 